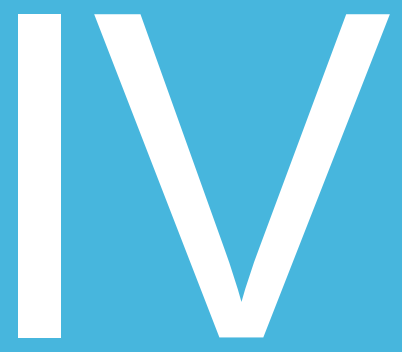

\title{
VIGENCIA DE LA CLASE
}

\section{MAGISTRAL EN LA UNIVERSIDAD DEL SIGLO XXI}

\section{Validity of the lecture at the University of the century - XXI Universidad Nacional del Altiplano}

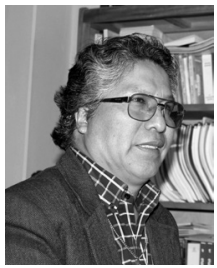

\section{Francisco Charaja Cutipa}

Bachiller en Periodismo. Licenciado en Educación y en Gestión Educativa. Magíster en Administración de la Educación. Doctor en Educación. Docente Principal de la Facultad de Ciencias de la Educación en la Universidad Nacional del Altiplano. Actualmente es docente de la asignatura de Administración Educativa en la Universidad Nacional del Altiplano. Docente de Metodología de la Investigación Científica, Didáctica Universitaria, Teorías de la Administración y Teoría de las Decisiones de los programas de Maestría y Doctorado en la Universidad Nacional del Altiplano. Docente itinerante en la Universidad Andina Néstor Cáceres Velásquez de Juliaca; Universidad Peruana Unión, sede Juliaca; Universidad Nacional Micaela Bastidas de Abancay; Universidad Inca Garcilaso de la Vega de Lima; Universidad San Francisco de Asís de la Paz-Bolivia; Universidad Andina del Cusco, entre otras. 


\section{Resumen}

El estudio se refiere a la estrategia de la clase magistral que es muy repudiada hoy día en los eventos curriculares por parte de supuestos expertos en didáctica o supuestos versados en teorías de la educación superior. Aquí se demuestra todo lo contrario. Las clases magistrales son efectivas, cuando se sabe implementar dentro del marco del aprendizaje significativo por recepción que propone Ausubel. Para sostener esta postura, se experimentó durante dos años académicos con estudiantes del VII semestre de la Escuela Profesional de Educación Secundaria de la Facultad de Ciencias de la Educación de la Universidad Nacional del Altiplano de Puno, Perú. Se trata de los estudiantes de la especialidad de Lengua y Literatura que llevaron la asignatura de Administración Educativa II en el año 2012-II y en el año 2013-I. La conclusión del experimento sostiene que las clases magistrales, cuando son interactivas y la exposición de la teoría es contextual, resultan eficaces para el logro de buenos niveles de aprendizaje. Además, en las clases magistrales interactivas, el dogmatismo y el monólogo expositivo son recusados de principio a fin.

Palabras clave: Aprendizaje, clase magistral, estrategia, interactivo.

\section{Abstract}

The study refers to the strategy of the Master Class is now repudiated in curricular events by suspected or alleged educational experts versed in theories of higher education. Here we demonstrate the opposite. The lectures are effective when implemented within the framework knows meaningful learning by Ausubel proposed reception. To sustain this position experienced in two academic years with students of VII semester of the Professional School of Secondary Education, Faculty of Education Sciences, National University of Altiplano de Puno, Peru. It is the specialty students Language Arts that brought the subject of Educational Administration II in 2012 - II and 2013 -I. The conclusion of the experiment argues that when lectures are interactive and when the exposition of the theory is contextual, are effective in achieving good levels of learning. In addition, interactive lectures, dogmatism and expository monologue are challenged from start to finish.

Keywords: Learning, lecture, strategy, interactive. 


\section{Introducción}

En la mayoría de los eventos académicos, donde se aborda el tema de la didáctica del docente universitario, se cuestiona la clase magistral calificándosela como una estrategia anacrónica, obsoleta y de origen conductista. Los supuestos expertos que abordan el tema no reparan que su acendrado cuestionamiento lo están realizando mediante una clase magistral, es decir, su exposición es una variante de la clase magistral, aunque mal ejecutada. La única diferencia es que utilizan el data displey, como para señalar que están con la tecnología de punta. O sea, lo moderno sería solo el uso de un equipo electrónico que proyecta los contenidos de la exposición, reemplazando a la técnica y al arte de escribir en la pizarra mientras se expone oralmente o a la técnica de utilizar papelógrafos para ayudarse durante la exposición oral. ¿Dónde está la diferencia de fondo o de esencia? No la hay en absoluto. Y, como si fuera poco, a estos supuestos expertos no les gusta que les cuestionen, o sea, no se dan cuenta que son más dogmáticos, conductistas y monocordes que el mismo papa.

La clase magistral, según deja entrever Certero (2001), es también denominada lección magistral o método expositivo. Es la estrategia didáctica más utilizada en los diferentes niveles educativos del mundo; pero, en la mayoría de las veces, mal implementada, lo que resultó en una mala reputación. Consiste en la exposición verbal de los contenidos de un tema o materia curricular. Morell (2004) señala, al respecto, que la clase magistral nace con el acto natural de enseñar del adulto al menor, del padre al hijo, del hermano mayor al hermano menor, del antiguo al nuevo, etc. Nadie enseña lo que no sabe o no domina, nadie enseña lo que no vivió o lo que no experimentó. Solo en este caso se da la enseñanza y, por lo tanto, la exposición es su medio natural. Por su parte Doménech (1999) señala que todo proceso de enseñanza-aprendizaje parte de la exposición oral del que sabe ante el que no sabe. Es decir, la comunicación oral del que sabe ante el que no sabe es un hecho concomitante a la naturaleza de la comunicación entre los seres humanos. En este siglo, como se sabe, la fuente de los conocimientos son los textos escritos y los textos digitales, lo que significa que la exposición está viva y más vigente que antes.

Por eso, no se puede prescindir, ahora ni en el futuro, de la exposición oral del que sabe ante el que no sabe. Esta es una verdad irrefutable. Pero el problema no queda ahí. El problema está en el hecho que el que expone puede hacerlo con la creencia de que lo que dice es la única verdad y, por lo tanto, el que lo escucha debe aceptarlo sin dudas ni murmuraciones; o puede exponer 
con la concepción de que lo que sabe es relativo y, además, el que lo escucha puede saber más que uno, es decir, no cree en la verdad dogmática. Por otro lado, el que parte de la premisa que sus conocimientos son dogmas, puede imponerlos verticalmente o sutilmente con una actitud del sabio que no quiere ponerse a discutir sus verdades con los "ignorantes". Así como puede darse el caso del que considera que sus conocimientos son relativos, pero evita el debate o las preguntas prefiriendo la recepción pasiva de sus alumnos o puede asumir la postura de un expositor abierto al debate, a las preguntas y a las críticas cognoscitivas. Este último caso es el tipo de docente del siglo XXI que aún sigue utilizando la clase magistral como una estrategia efectiva durante el proceso de la enseñanza-aprendizaje universitario.

Certero (2001) deja entender que la clase magistral del siglo pasado, cuestionada y recusada en la literatura didáctica actual como negativa para el aprendizaje y formación de los estudiantes universitarios, sea hoy reivindicada como estrategia muy adecuada para la enseñanza superior, pero con la característica de que la exposición sea acompañada por auxiliares audiovisuales y esté abierta permanentemente al debate, a las preguntas y a los cuestionamientos cognoscitivos. A esta estrategia es a la que Morell (2004) denomina la clase magistral interactiva. Por eso, se puede concluir que la clase magistral del pasado era unidireccional, con lo que no compartimos en este artículo. Aquí apostamos por la clase magistral interactiva que, por esencia, es una comunicación bidireccional o coexpositiva.

La Clase Magistral Interactiva, según los autores consultados y la postura que el autor asume, consiste en tres pasos básicos: Introducción, desarrollo y término. La introducción es la presentación del tema relacionándolo con la realidad coyuntural. El desarrollo es la exposición secuencial y sistemática del docente con el auxilio de una de las técnicas ideográficas, por un lado, y, por otro lado, es la exposición de los estudiantes en forma de preguntas, objeciones, opiniones o cuestionamientos cognoscitivos por iniciativa propia o por la motivación didáctica del docente. El término es una especie de resumen que generalmente se debe realizar con la participación directa de los estudiantes, es decir, quienes deben resumir son los estudiantes. La condición sine qua non de esta estrategia es la premisa de que no existe la verdad absoluta y que el docente no es el depositario de la verdad del conocimiento.

El tipo de aprendizaje que se promueve, con la clase magistral interactiva, es el aprendizaje por recepción planteado por Ausubel (2002). Este tipo de aprendizaje entra en contradicción con el aprendizaje por descubrimiento que plantea Piaget y Brunner, y contra todos aquellos que apuestan por el 
aprendizaje activo o por el aprendizaje manipulativo. Como sostiene Ausubel (2002) si todo lo que hay que conocer durante nuestra corta existencia, tenemos que aprender descubriendo de nuevo, manipulando o experimentando, no llegaríamos a conocer lo que debemos saber, ni tampoco avanzaría la ciencia ni la tecnología como avanza hoy. Por tanto, es absurdo pretender cuestionar la estrategia de la clase magistral interactiva o bidireccional. Todo lo contrario, esta estrategia es ineludible en la enseñanza universitaria. Por supuesto que en las materias en las que se deben trabajar en laboratorios, llegado el momento didáctico, se tiene que experimentar y se tiene que replicar. Tal como sucede con todas las demás ciencias. Aquí no debemos olvidar que la verdad del conocimiento se tiene que encontrar en la práctica de la teoría expuesta o recibida para que, luego, esta práctica enriquezca la teoría, y así sucesivamente.

\section{Antecedentes}

Villanueva (2011) realizó un experimento aplicando el método de la clase magistral para ver qué niveles de rendimiento académico logran los estudiantes de la Carrera de Secretariado Ejecutivo. En este estudio concluye que la prueba de rendimiento, aplicada a los estudiantes de la asignatura de Análisis de la Realidad Peruana, da como resultado que el $80 \%$ de los estudiantes del grupo experimental, obtuvo un rendimiento académico alto y $20 \%$, un rendimiento medio. Asimismo, en los estudiantes del grupo de control, el $53 \%$ tuvo un rendimiento medio, $37 \%$, un rendimiento bajo y solo el $10 \%$, un rendimiento alto.

En otra tesis de licenciatura, Escudero (2008) realiza una investigación experimental referida a la eficacia del método expositivo, asistido por modelos interactivos. En el estudio se concluye que el método expositivo, asistido por un modelo interactivo utilizando ordenadores, fortalece y complementa los dominios del aprendizaje. Del análisis realizado, se deduce que las habilidades medidas mejoraron de un promedio de 8,74 (antes) hasta un promedio de 10, 76 puntos (después).

Tarabay (2003), en su tesis doctoral referida a las estrategias argumentativas en el discurso docente de la clase magistral universitaria, concluye que cuando la clase magistral del docente es adecuadamente argumentada, de por sí se genera el diálogo o la participación de los estudiantes. Esta técnica es efectiva, sobre todo, en aquellas materias de carácter teórico. 


\section{Materiales y métodos}

Ámbito de estudio: El estudio se realizó en la Escuela Profesional de Educación Secundaria de la Facultad de Ciencias de la Educación de la Universidad Nacional del Altiplano de Puno, Perú, Especialidad de Lengua y Literatura, durante los años académicos 2012-II y 2013-I.

Población y muestra: La población de estudio estuvo constituida por los estudiantes de la especialidad mencionada que, en los dos años académicos señalados, Ilevaron la asignatura de Administración Educativa II. En el año 2012-II se matricularon en la asignatura 23 estudiantes; mientras que en el año 2013-I lo hicieron 24 estudiantes.

Descripción de técnicas e instrumentos de investigación: Para recoger los datos correspondientes a los objetivos propuestos, se utilizó solo la técnica del examen y su instrumento fue la prueba de conocimientos de tipo objetivo. Las pruebas fueron formuladas con mucho cuidado, considerando los criterios de validez y confiabilidad. Cada prueba consistió en 10 preguntas relacionadas con los contenidos desarrollados.

Frecuencia temporal de recojo de datos: En cada semestre se aplicaron en dos oportunidades las pruebas de conocimientos, correspondientes en cada caso a una unidad didáctica.

Prueba estadística de la hipótesis: Dado que solo se quiere determinar cuánto mejora el aprendizaje de los estudiantes con la estrategia de las clases magistrales, se consideró solo la media aritmética como la prueba estadística de la hipótesis central.

\section{Resultados y discusiones}

El principal objetivo planteado, en el estudio, fue la determinación del nivel de aprendizaje promedio que la mayoría de los estudiantes obtuvieron al cabo del semestre académico en la asignatura de Administración Educativa II. Se debe señalar que, durante el semestre académico, la enseñanza de la asignatura se realizó con la estrategia de la clase magistral interactiva.

Tal como se observa en la tabla 1, la mayoría de los estudiantes del VII semestre de la especialidad de Lengua y Literatura obtuvo calificativos com- 
prendidos entre 14 y 17 puntos, lo que significa que la mayoría (56\%) obtuvo buenos niveles de aprendizaje. Solo 2 estudiantes (9\%) fueron desaprobados. La media aritmética que se obtiene es de 15 puntos, es decir, el calificativo promedio de la mayoría de los estudiantes corresponde al nivel bueno.

\section{Tabla 1}

Niveles de aprendizaje en la asignatura de Administración Educativa II, 2012-II

\begin{tabular}{llcl}
\hline \multicolumn{2}{c}{ NIVELES DE APRENDIZAJE } & $\mathbf{f}$ & \% \\
\hline Aprendizaje muy deficiente & $01-05$ & 00 & 00 \\
Aprendizaje deficiente & $06-10$ & 02 & 09 \\
Aprendizaje regular & $11-13$ & 05 & 22 \\
Aprendizaje bueno & $14-17$ & 13 & 56 \\
Aprendizaje muy bueno & $18-20$ & 03 & 13 \\
Total & & 23 & 100 \\
\hline
\end{tabular}

Fuente propia

$$
\bar{X}=\frac{\sum(X i)(f)}{n} \quad \bar{X}=\frac{334.5}{23}=15
$$

En la tabla 2 se muestran los resultados del aprendizaje que los estudiantes lograron en el I semestre del año académico 2013. En este cuadro se observa que 14 estudiantes (58\%) obtuvieron calificativos comprendidos entre 14 y 17 puntos, lo que corresponde al nivel bueno. Solo 3 estudiantes fueron desaprobados con calificativos de 08,09 y 10 puntos respectivamente. La media aritmética que corresponde es de 14 puntos, lo que confirma la tendencia de los estudiantes, en cuanto al nivel de aprendizaje predominante.

\section{Tabla 2}

Niveles de aprendizaje en la asignatura de Administración Educativa II, año 2013-I

\begin{tabular}{cccc}
\hline \multicolumn{2}{c}{ NIVELES DE APRENDIZAJE } & f & $\%$ \\
\hline \multicolumn{2}{c}{ Aprendizaje muy deficiente 01-05 } & 00 & 00 \\
Aprendizaje deficiente & $06-10$ & 03 & 13 \\
Aprendizaje regular & $11-13$ & 05 & 21 \\
Aprendizaje bueno & $14-17$ & 14 & 58 \\
Aprendizaje muy bueno & $18-20$ & 02 & 08 \\
Total & & 24 & 100 \\
\hline
\end{tabular}

Fuente propia 


$$
\bar{x}=\frac{339}{24}=14
$$

Los resultados de ambos cuadros, correspondientes al aprendizaje en la asignatura de Administración Educativa II, confirman la hipótesis que se planteó en esta investigación. En el estudio se planteó que los niveles de aprendizaje que la mayoría de los estudiantes obtienen en el semestre, recibiendo las clases magistrales interactivas, es correspondiente al nivel bueno. Las medias aritméticas de 15 puntos en el año 2012 y de 14 puntos en el año 2013, confirmando la eficacia de la estrategia de las clases magistrales interactivas. Por otro lado, se confirma en el mismo sentido los resultados de las investigaciones que se consideran como antecedentes. Este es el caso de Villanueva (2011) quien, en su estudio, concluye que el rendimiento en la asignatura de Análisis de la Realidad Peruana mejora con las clases magistrales. De la misma manera, en el estudio realizado por Tarabay (2003), se concluye que cuando la clase magistral del docente es adecuadamente argumentada, de por sí se genera el diálogo o la participación de los estudiantes y los aprendizajes mejoran.

La teoría existente acerca de las clases magistrales, en el sentido que esta estrategia privilegia el aprendizaje por recepción (Ausubel, 2002), solo será efectiva en la medida que el docente parta de la premisa que no es el depositario del saber absoluto y que su exposición oral sea auxiliada por técnicas ideográficas, por la lectura, por el diálogo, por las preguntas y por el cuestionamiento cognoscitivo de parte del estudiante.

Los resultados de esas investigaciones permiten afirmar que es una estrategia muy efectiva, especialmente en las materias relacionadas con las teorías sociales como pedagogía, filosofía, administración, historia, etc. Sin embargo, la clase magistral es negativa e ineficaz cuando el profesor asume la premisa dogmática de que él es el depositario del saber absoluto y que sus alumnos solo deben escucharle pasivamente sin ningún tipo de cuestionamientos ni preguntas. La exposición unidireccional y dogmática es la técnica que socavó las bases de la efectividad de una clase magistral didácticamente implementada, como se pudo aplicar en este experimento realizado en los dos años académicos señalados.

Por consiguiente, como conclusión principal de esta investigación, se asume que las clases magistrales interactivas son eficaces para el logro de buenos niveles de aprendizaje si se implementa dentro de la tecnología del 
foro abierto y de la comunicación bidireccional y, además, cuando la exposición de la teoría es contextual y motivadora. En las clases magistrales interactivas, el dogmatismo y la exposición monocorde deben ser recusados de principio a fin.

Francisco Charaja Cutipa Universidad Nacional del Altiplano, Perú email: charaja1958@hotmail.com

Recibido: 7 de enero de 2014 Aceptado: 30 de marzo de 2014 
- Francisco Charaja Cutipa

\section{Referencias}

Ausubel, D.P. (2002). Adquisición y retención del conocimiento. España: Paidós.

Biggs, J. (2006). Calidad del aprendizaje universitario. Madrid: NARCEA.

Certero, A.M. y otros (2001). El discurso académico oral: guía didáctica para la comprensión auditiva y visual de clases magistrales. Madrid: Edinumen.

Charaja, F. (2012). Ingeniería de la educación. Puno: Sagitario Impresores.

Doménech, F. (1999). El proceso de enseñanza-aprendizaje universitario. España: Universitat Jaume I.

Escudero, G. S. (2008). El Método expositivo asistido por ordenadores utilizando modelos interactivos en la enseñanza universitaria (Tesis de Licenciatura). Universidad Nacional Mayor de San Marcos, Lima, Perú.

Morell, T. (2004). La interacción en la clase magistral. España: Universidad de Alicante.

Tarabay, F. (2003). Estrategias argumentativas en el discurso docente de la clase magistral universitaria (Tesis Doctoral). Universidad de Los Andes, Mérida, Colombia.

Villanueva, O.L. (2011). El método de la clase magistral en el rendimiento académico de los estudiantes de la asignatura de Análisis de la Realidad Peruana, II Semestre de Secretariado Ejecutivo, IST Joaquín Reátegui Medina del distrito de Nauta, 2009 (Tesis de Licenciatura). Universidad Nacional Mayor de San Marcos, Lima, Perú. 